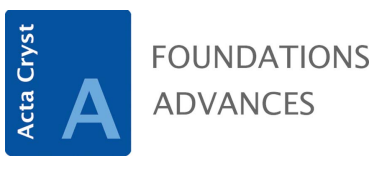

ISSN 2053-2733

\section{Introduction to Many-Body Physics. By Piers Coleman. Cambridge University Press, 2015. Pp. xviii + 796. Price GBP 49.99/USD 84.99 (hardback). ISBN 9780521864886.}

\author{
Sébastien Lebègue* \\ Université de Lorraine, CNRS, LPCT, Nancy, France. *Correspondence e-mail: sebastien.lebegue@univ-lorraine.fr
}

A knowledge of many-body physics is essential to any graduate student or researcher who wants to understand the quantum laws governing the properties of matter. The book Introduction to Many-Body Physics by Piers Coleman aims to provide its readers with a solid background in this field, which then should allow them to turn to more specialized volumes or research publications.

The first chapter, entitled 'Scales and complexity', reminds the reader of the fact that the Schrödinger equation is impossible to solve in a general case, and introduces the concept of microscopic and macroscopic scales divided into four main categories: time scale, length scale, particle number and complexity. The second chapter, under the title 'Quantum fields', introduces second quantization and treats in detail the one-dimensional harmonic oscillator using this formalism. As expected, this is followed by a section on phonons, while the two last parts of this chapter revisit the previous developments in the light of the thermodynamic and continuum limits. The third chapter ('Conserved particles') goes deeper into the formalism of quantum-field theory and provides the reader with the necessary knowledge on commutation and anticommutation rules, creation and annihilation operators, vacuum, and on the second quantized representation of the interaction between particles, which allows one to rewrite the Schrödinger equation. The fourth chapter is built upon the two previous ones and presents several well known examples using second quantization, such as the Jordan-Wigner transformation with an application to the one-dimensional Heisenberg mode, the Hubbard model, and a paragraph on non-interacting bosons and fermions in thermal equilibrium, in which the BoseEinstein condensation is introduced. These four chapters constitute all together the minimum basis for understanding the rest of the book.

In the fifth chapter, the concept of Green's functions is introduced with the help of the interaction representation. An experienced reader will find here what they expect: the time-ordering operator, Wick's theorem and the Green's function of free particles. Then the concept of adiabatic switching is detailed, followed by a short section on manyparticle Green's functions. The sixth chapter, of nearly 50 pages, presents Landau's theory of the Fermi liquid. As expected for a textbook on this topic, the concept of quasiparticle is introduced and a detailed comparison between the non-interacting and the interacting liquids is made. However, the author added to this standard matter several paragraphs on scattering phenomena, collective modes and charged Fermi liquids, topics that are usually not found in introductory books.

In Chapter 7, Feynman diagrams at zero temperature are presented. The different rules in real and momentum spaces are summarized and several examples are given, such as the Hartree-Fock energy or the concept of exchange correlation. Then, the self-energy is presented and particular attention is paid to the random-phase approximation for the electron gas. Logically, the following chapter is about finite-temperature many-body physics. It starts with the imaginary time formalism and the Matsubara representation, and revisits Feynman rules and Feynman diagram expansion. The chapter ends with almost 20 pages on electron-phonon interactions.

Chapter 9 is devoted to the fluctuation-dissipation theorem and linear-response theory. The formalism is introduced in the case of the classical harmonic oscillator and then generalized to the quantum case with a link to response functions. The last part of the 
chapter directly targets experimentalists and deals with electron, spin and transport spectroscopies.

The tenth chapter is quite short (about 20 pages) and is devoted to electron-transport theory in the framework of Green's functions and Feynman diagrams. The Kubo formula, Drude theory of conductivity and Anderson localization are presented. The 11th chapter deals with phase transitions and broken symmetry: the signification of the order parameter is explained, then Landau and Landau-Ginzburg theories are detailed. This last point is developed further in three large parts and therefore covers most of what the reader should know about it before turning to more specialized publications. Then, the chapter further develops the topics of the Anderson-Higgs mechanism and criticality.

Chapter 12 concerns path integrals, something which is expected in any book on many-body theory. Here the author delves deep (more than 40 pages) into the details of the formalism: the principle of path integrals and coherent states are presented first, followed by some notions of Grassman algebra (which are completed by several appendices) and finally the Hubbard-Stratonovich transformation. The 13th chapter bears the title 'Path integrals and itinerant magnetism'; it is directly linked to the previous one and focuses on itinerant magnetism. The Stoner criterion, which defines the condition for a system to have a finite spin polarization, is presented, followed by the Hubbard model formulated in terms of path integrals. Finally, a paragraph is devoted to the mean-field theory of magnetism and the chapter is concluded with some text on quantum magnetic fluctuations.

Chapter 14 concerns a different area of condensed-matter physics, namely superconductivity. This extensive chapter (more than 50 pages) begins with several pages on the Cooper instability, naturally followed by a presentation of the BCS Hamiltonian and the pairing terms. Then, the NambuGor'kov Green's function is detailed and the stiffness of the phase of a superconductor is discussed. The next chapter, 'Retardation and anisotropic pairing', complements the previous one: the BCS theory with momentum-dependent coupling is presented and $d$-wave pairing in two dimensions, as it is related to cuprate superconductors, is highlighted. The chapter ends with a discussion on superfluid helium.

Chapter 16 introduces the reader to the field of strongly correlated systems. The concept of local magnetic moments is presented, followed by the well known Anderson's model. Then the phenomenon of Coulomb blockade is briefly summarized, followed by a large part on Kondo physics and Nozières-Fermi liquid theory. Chapter 17, entitled 'Heavy electrons', is on the same topic as the previous one but with a focus on heavy-fermion states. The RKKY interaction is presented, followed by the Coqblin-Schrieffer model. Then, mean-field theories of the Kondo impurity and of the Kondo lattice are detailed, followed by several pages on Kondo insulators, tunnelling in heavy-electron fluids and optical properties of systems with heavy electrons. The last chapter, under the title 'Mixed valence, fluctuations, and topology', covers certain aspects that were not discussed in the two previous chapters: for instance, the slave boson method, spin and valence fluctuations, and topological Kondo insulators.

With each chapter comes a series of examples and exercises, although the difference between the two is not obvious, except that the exercises are found at the end of the chapter while the examples appear in the course of the text, which I found a bit distracting. Also, for no clear reason, solutions are provided for the examples but not for the exercises.

The book ends with a short epilogue ('The challenge of the future') in which the author proposes several directions to follow for future progress in the field. Here I regret that density-functional theory and multi-determinant wavefunction methods are not even mentioned, despite the important achievements that have been reached over the last 20 years, and the developments that are still going on. In the same way, $a b$ initio calculations using the random-phase approximation, the GW approximation or the Bethe-Salpether equation, which are Green's function methods, are sparking intense interest as they become more affordable thanks to several recent theoretical and computational improvements, and would have deserved their own paragraph here.

Apart from that, this book is excellent and will nicely complement the well known standard volumes by Mattuck (1992), Mahan (2000) and Fetter \& Walecka (2003). It will also update the reader with some recent developments, something that older books obviously cannot do, although in my opinion these developments fall beyond a mere introduction to manybody physics. Also, I liked the way the author has put the various theories or methods in their historical context, which certainly makes the book more pleasant to read and even more informative. In conclusion, I recommend this book without hesitation.

\section{References}

Fetter, A. L. \& Walecka, J. D. (2003). Quantum Theory of ManyParticle Systems. New York: Dover.

Mahan, G. D. (2000). Many-Particle Physics, 3rd ed. Dordrecht: Springer.

Mattuck, R. D. (1992). A Guide to Feynman Diagrams in the ManyBody Problem. New York: Dover. 